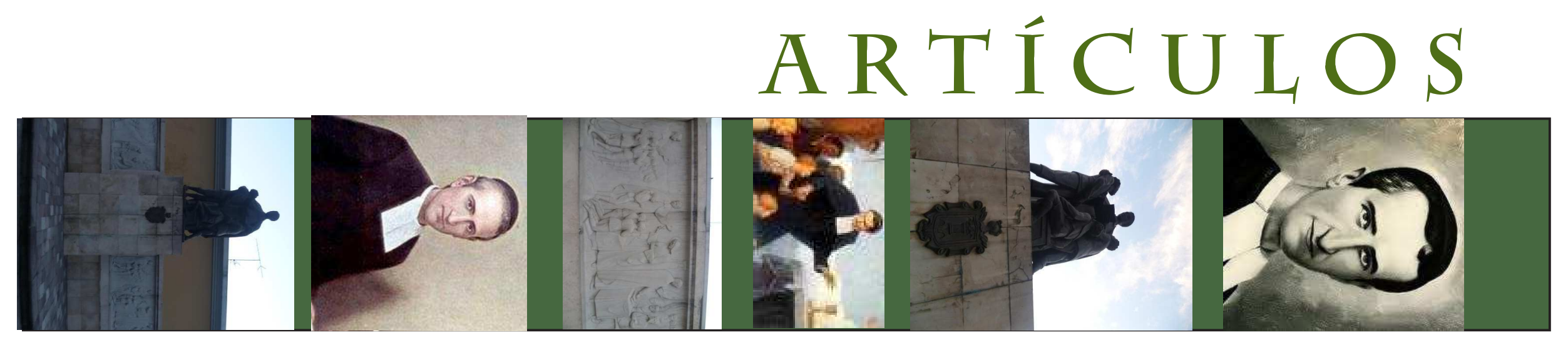




\title{
G.M. BRUÑO SAN MIGUEL FEBRES CORDERO E1 Hermano Cristiano de los Textos Escolares
}

\author{
Javier Ocampo López ${ }^{1}$ \\ Academia Colombiana de Historia - Colombia \\ Grupo de investigación HISULA \\ javierocampo11@yahoo.es
}

Recepción: 27/04/2011

Evaluación: 09/05/2011

Aceptación: 22/06/2011

Artículo de Reflexión

\section{RESUMEN}

El principal objetivo de este texto es el estudio sobre la vida, el pensamiento y la acción del Hermano Cristiano G.M. Bruño, San Miguel Febres Cordero, uno de los autores de textos escolares de mayor influencia en Hispanoamérica desde finales del siglo XIX y en la primera mitad del siglo XX. Se recuerdan los textos de Aritmética, Álgebra y Geometría del Hermano Cristiano G.M. Bruño, que formaron a los colombianos, ecuatorianos y de otros países de Hispanoamérica. Escribió más de cincuenta textos de lenguaje español y literatura, ortografía, física, botánica, religión, historia sagrada, tabla de logaritmos, contabilidad, y otros. La Colección de Textos de G.M. Bruño es una de las más variadas y de trascendencia en la Textología de Hispanoamérica.

Palabras clave: Revista Historia de la Educación Latinoamericana, textos escolares, G.M. Bruño, comunidad hermanos cristianos, pedagogía católica, lasallismo.

\footnotetext{
1 Doctor en Historia, es profesor del Doctorado en Ciencias de la Educación RUDECOLOMBIA, miembro de la Academia Boyacense de Historia y Academia Colombiana de Historia y del grupo de investigación Historia y Prospectiva de la Universidad Latinoamericana - HISULA. Este trabajo hace parte del proyecto "La independencia americana: textos e imaginarios escolares en Colombia y España" DIN - UPTC, código SGI 772, grupos de investigación ILAC e HISULA.
} 


\title{
G.M. BRUÑO \\ SAN MIGUEL FEBRES CORDERO \\ The Christian Brother of scholar texts
}

\author{
Javier Ocampo López \\ Academia Colombiana de Historia - Colombia \\ Research Group HISULA \\ javierocampo11@yahoo.es
}

\begin{abstract}
The main goal of this text is the study about life. The Christian brother`s thought and action G.M. Bruño, San Miguel Febres Cordero. He is one of the scholar texts authors with the highest influence on Latin America at the end of century XIX and in the first half of XX. Arimetic, Algebra and geometrics texts from Christian Brother G.M. Bruño are remembered; those texts created Colombian and Ecuatorian people and others from other Latin American countries. He wrote more that fifty texts in Spanish and Literature, Spelling, Physics, Botany, Religion, Sacred History, Logarithm Chart, Accounting among others. The collection texts from G.M. Bruño is one of the most diverse and of importance in the Latin America Textology.
\end{abstract}

Key words: Journal of Latin American Education History, scholar texts, christian brothers, catolic pedagogic and lasallian. 


\title{
G.M. BRUÑO SAN MIGUEL FEBRES CORDERO O Irmão Cristão dos textos escolares
}

\author{
Javier Ocampo López \\ Académica Colombiana de Historia - Colombia \\ Grupo de pesquisa HISULA \\ javierocampo11@yahoo.es
}

\section{RESUMO}

O principal objetivo deste texto é o estudo da vida, pensamento e atividade do Irmão Cristão G.M. Bruño, San Miguel Febres Cordero, um dos autores de textos escolares de maior influência na América Hispânica desde finais do século XIX e na primeira metade do século XX. Recordamos os textos de Aritmética, Álgebra e Geometria do Irmão Cristão G.M. Bruño, que formaram os colombianos, equatorianos e pessoas de outros países da América Hispânica. Escreveu mais de cinquenta textos de língua espanhola e literatura, ortografia, física, botânica, religião, história sagrada, tábua de logaritmos, contabilidade, e outros. A Coleção de textos de G.M. Bruño é uma das mais variadas e transcendentes na Textologia da América Hispânica.

Palavras- chave: Revista História da Educação Latino-americana, textos escolares, G.M. Bruño, comunidade irmãos cristãos, pedagogia católica, lasallismo. 


\section{INTRODUCCIÓN}

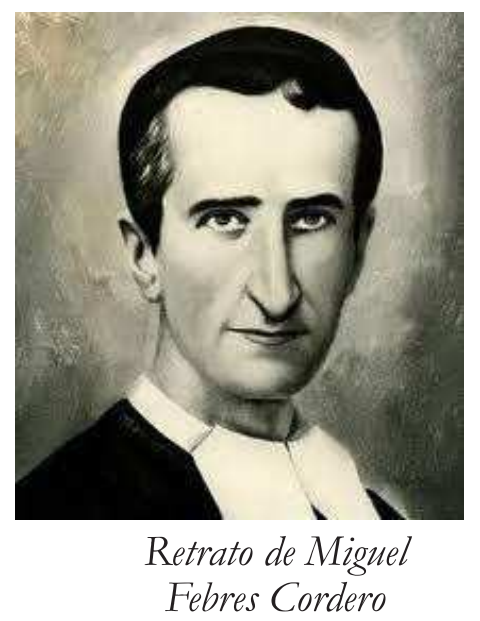
otros lugares del mundo. Por sus méritos religiosos fue beatificado por el Pontífice Paulo VI y llevado a los altares como San Miguel Febres Cordero por el Papa Juan Pablo II.

Los textos escolares, como los de la Colección G.M. Bruño del educador ecuatoriano San Miguel, son importantes como instrumentos auxiliares en la educación. Hacen parte del material didáctico para las clases en las aulas. El texto es un compendio auxiliar de estudios para uso de los alumnos. Es un instrumento orientador de la enseñanza, auxiliar, didáctico en las prácticas y ejercicios. Los textos son de significativa importancia para el planeamiento de 2 clases del profesor y para la orientación de estudios de los alumnos.

Algunos textos escolares sirven para perpetuar los métodos y contenidos tradicionales; y otros más modernos proyectan los nuevos cambios en la pedagogía. Los textos proporcionan a los profesores y estudiantes materiales que pueden utilizar para la enseñanza y el aprendizaje. Generalmente estos textos escolares se basan en la experiencia de sus autores. Proporcionan problemas y ejercicios complementarios y sugieren actividades para los alumnos. Ayudan a organizar los materiales del curso, tanto para los profesores como para los estudiantes.

\footnotetext{
${ }^{2}$ Véase la obra de IMIDEO G., Nerici. (1969): Hacia una Didáctica General Dinámica. Buenos Aires, Editorial Kapeluz, pp. 325-338.
} 
Los textos escolares son una buena fuente de consulta para usar en el esbozo de los temas o en los problemas que se sugieren para el aprendizaje. Sirven para la descripción de los temas en síntesis, los problemas y otros aspectos didácticos, siguiendo los programas escolares de las autoridades educativas. También sirven de ayuda a la consulta para obtener datos u otro tipo de ayuda. Muchos profesores utilizan los textos escolares para enseñar a estudiar a sus alumnos y para utilizar los libros. Los textos escolares organizan los contenidos con exactitud y son fundamentales para la organización de la enseñanza: los objetivos, el desarrollo de los temas, los métodos instructivos que se utilizan en el texto y las investigaciones que se deben realizar para complementar el proceso enseñanza-aprendizaje.

Los textos escolares están fundamentados en un lenguaje científico accesible al nivel intelectual de los alumnos a los cuales está destinado. Deben ofrecer síntesis, resúmenes, lecturas, problemas, indicaciones bibliográficas relativas a los asuntos estudiados. Deben contener motivaciones e indicaciones para la ampliación del aprendizaje.

En la metodología de la investigación científica y de divulgación, se tienen en cuenta los procedimientos para la elaboración de las obras didácticas o textos escolares. Un manual didáctico debe tener una visión completa y sistemática de la materia que va a tratar. Se señala que el texto es un guía, una base general para el conocimiento de las ciencias naturales, físicas, matemáticas, ciencias sociales y otras. En su elaboración se tiene en cuenta la sistematización, la síntesis y el conjunto. Los autores de textos obtienen los conocimientos de las obras de los grandes científicos, las cuales sintetizan para llevarlas a los estudiantes a través de los textos escolares. Tienen la característica de labor didáctica a través de la síntesis del conjunto de una ciencia para entregarla progresivamente a los estudiantes mediante pasos o etapas.

El Hermano Cristiano G.M. Bruño, San Miguel Febres Cordero, en sus estudios e investigaciones aportó a la textología escolar, siguiendo las orientaciones de la Pedagogía Católica que ha tenido importancia en

\footnotetext{
${ }^{3}$ Para el estudio de los temas educativos y textos escolares, véase la obra de NOLEN y GOETZ. (1961): Prontuario del Escritor de Materias educativos. México, Editorial PaxMéxico, pp. 102-114. Asimismo, la obra de LASSO DE LA VEGA, Javier. (1975): El Trabajo intelectual. Normas, técnicas y ejercicios de documentación. Madrid, Paraninfo.
} 
la educación hispanoamericana desde el siglo XVI. El Imperio Español, "Adalid del Catolicismo" a nivel mundial hizo la transculturación política, socio-económica, cultural y religiosa en Hispanoamérica, la cual se proyectó a la evangelización, la formación misional y la educación católica de los pueblos aborígenes del Nuevo Mundo o América. Esta pedagogía la continuó España en los siglos XVI, XVII y XVIII, en la época colonial y se continuó en la vida republicana en los siglos XIX, XX y XXI.

Después de la Revolución de Independencia, la Iglesia Católica tuvo enfrentamientos con el Liberalismo en Hispanoamérica. El Radicalismo señaló la necesidad de la separación entre la Iglesia y el Estado, la educación laica, el divorcio y la no interferencia del Clero en las actividades políticas. En la segunda mitad del siglo XIX se manifestaron tendencias políticas hacia la unidad entre la Iglesia y el Estado; y en especial, la influencia de la Iglesia en la educación. Así ocurrió en Colombia con el Movimiento de la Regeneración en la década de los ochenta que llevó al Concordato de 1887, en las relaciones entre la Iglesia y el Estado y en Ecuador, en el Gobierno del Presidente Gabriel García Moreno, quien gobernó entre 1859 y $1875^{4}$.

En los gobiernos de derecha de estos países se introdujeron las Comunidades religiosas para la orientación y dirección de la educación, entre ellas la Comunidad de los Hermanos Cristianos, los Hermanos Maristas, la Compañía de Jesús, los Padres Salesianos, los Padres Lazaristas, los Padres Eudistas, las Hermanas Dominicas de la Presentación, las Betlehemitas o Hijas del Corazón de Jesús y otras comunidades que influyeron en la educación y en la solución a los problemas de la salud.

\section{La pedagogía católica de los hermanos cristianos}

Esta Comunidad de educación católica fue fundada por San Juan Bautista de La Salle (1651-1719), natural de Reims (Francia), de ilustre familia, oriunda de España. Sacerdote, formado en Saint-Sulpice, doctor en Teología. En 1679 fundó dos escuelas de caridad para niños pobres que fueron el punto de partida para la creación de la Comunidad de los Hermanos de las Escuelas Cristianas, fundada en el año 1682,

\footnotetext{
${ }^{4}$ Véase la obra de PONCE LEIVA, Pilar. (1987): Gabriel García Moreno. Madrid, Ediciones Quórum. Sobre los temas políticos y culturales de Ecuador en el siglo XIX, véase la obra del historiador NÚÑEZ SEGURA, Jorge. (2003): El Ecuador en el siglo XIX. Quito, CCE Benjamín Carrión.
} 
dedicada a la educación cristiana de los niños pobres. Por ello fue su interés la fundación de numerosas escuelas gratuitas para el pueblo. En su filosofía educativa instituyó la enseñanza individual, sustituyendo la enseñanza colectiva. Escribió su libro "Regla de meditaciones" para uso de los Hermanos Cristianos. Además, escribió varios libros con temas pedagógicos, entre ellos: Conduite des écoles (1706), la obra Letres, las Meditations. Sus obras: "Guia de las escuelas", "Reglas de cortesía y urbanidad cristiana", "Colección de diferentes tratados", "Meditaciones sobre el ministerio de la enseñanza" y otras.

San Juan Bautista de La Salle fue el educador más representativo de la pedagogía popular francesa de los siglos XVII y XVIII, en torno al maestro, la escuela y la educación para los niños pobres. La Salle se dedicó especialmente a la educación de las clases populares. Se interesó por formar los maestros, quienes son esenciales para la formación de las jóvenes generaciones. Organizó Seminarios para los maestros de las escuelas, los cuales se convirtieron en verdaderas Escuelas Normales. Organizó diversos tipos de Escuelas: escuelas elementales, escuelas secundarias modernas, escuelas del domingo, escuelas técnicas, escuelas de reforma para los inadaptados, e institutos para la formación de religiosos.

Las Escuelas de San Juan Bautista de La Salle eran gratuitas para las gentes pobres o de escasos recursos. En ellas se enseñaba religión, rudimentos de lectura y escritura, nociones de aritmética y otras materias de provecho para la vida. Se interesó por enseñar uno o más oficios, pues su filosofía educativa era la instrucción práctica. Se organizaron escuelas dominicales para obreros y se crearon instituciones correccionales para los niños y jóvenes desadaptados. Para la formación de maestros calificados fundó seminarios pedagógicos o escuelas normales para maestros laicos, pues los Hermanos Cristianos se formaron en su Comunidad, con un objetivo de enseñanza para las gentes pobres, con educación gratuita y con interés por la instrucción práctica y técnica alrededor de los oficios.

San Juan Bautista de La Salle murió en Rouen (Francia) en el año 1719, dejando la Comunidad de los Hermanos Cristianos con 274 religiosos que se expandieron por todo el mundo, especialmente en

${ }^{5}$ Sobre San Juan Bautista de La Salle, véase su obra Guía de las Escuelas Cristianas (Manuscrito). Edición Príncipe de 1720. Distrito Lasallista de Perú.

ABBAGNANO, N. y VISALBERGHI, A. (2001): Historia de la Pedagogía. México, Fondo de Cultura Económica, 2001, pp. 312-313. Véase asimismo la obra de MANUEL MORENO, Juan y otros. (1986): Historia de la Educación. Madrid, Paraninfo, pp. 249-253. 
Europa y América. Su mayor interés fue la consolidación de la escuela popular gratuita y obligatoria para las clases pobres. Fue canonizado en 1900; y en 1951, el Pontífice Pío XII lo proclamó patrón de los educadores católicos, cuya fiesta es el 15 de mayo.

La pedagogía Lasallista de los Hermanos Cristianos se interesó desde un principio por la enseñanza popular y dio importancia a la formación de los maestros, creando las primeras escuelas normales laicas. Su interés fue la educación gratuita para los niños pobres y las escuelas dominicales o Academias cristianas para jóvenes obreros, con educación práctica, con cursos de contabilidad, arquitectura, geometría, dibujo, etc. Por ello su interés fueron las escuelas técnicas con educación práctica para ciertos oficios. Se fundaron las primeras instituciones para la formación de "Bachilleres técnicos".

Los Hermanos Cristiano establecieron los Reformatorios para niños indisciplinados y difíciles. Para su formación se aplicó una escuela de artes y oficios para que los niños de problemas no se formaran en prisión, sino con una educación práctica. Se aplicó una pedagogía diferencial, los débiles, los medianos y los fuertes. Al frente de cada clase se encomendó un Hermano cristiano, quien establecía la disciplina y el orden escolar.

En la pedagogía lasallista se dio importancia a la instrucción y la religión. La ignorancia es la fuente de todos los males, por lo cual hay que combatirla con la educación. La escuela se convierte en el noviciado de la vida, en donde se forma el hombre y el cristiano. Se aplica el modelo pedagógico del interrogatorio, pues es importante que los alumnos se den cuenta del sentido de las palabras y de los períodos.

La pedagogía lasallista busca la formación de los educandos con un currículo fundamentalmente práctico y concreto. La educación moral se fundamenta en la religión, que a su vez es la base de todas las virtudes cristianas. Referente a la disciplina, la pedagogía lasallista busca la prevención antes de acudir a la represión. La educación lasallista dio importancia a la lengua materna en la enseñanza primaria popular, con exclusión absoluta del latín. Se consideró que la lengua materna está más cerca de los intereses de los educandos. En sus primeras escuelas 
en Francia, San Juan Bautista de La Salle ordenó enseñar a los niños la lectura del francés desde su ingreso en la escuela .

La Comunidad de los Hermanos Cristianos entró a Hispanoamérica por Ecuador, cuando el Presidente Gabriel García Moreno la introdujo en 1863. Posteriormente los Hermanos Cristianos entraron a Colombia procedentes de Ecuador en los años del Movimiento de la Regeneración en las últimas décadas del siglo XIX. Bajo los auspicios de Monseñor Bernardo Herrera Restrepo, Obispo de Medellín ingresaron a Colombia seis Hermanos Cristianos, cuyo objetivo fundamental fue establecer la primera obra de la Comunidad de los Hermanos Cristianos en Medellín, el Colegio San José, fundado el 9 de abril de 1890. Cuando Monseñor Herrera fue nombrado Arzobispo de Bogotá en el año 1891, estableció la Comunidad de los Hermanos Cristianos en la capital, quienes fundaron el Instituto de La Salle en 1896. Posteriormente, los Hermanos Cristianos fundaron en Barranquilla el Colegio San José, que luego fue el Colegio Biffi. También se fundó un Instituto de los Hermanos de las Escuelas Cristianas en Honda. Se organizaron los Colegios, las Escuelas cristianas gratuitas para los niños pobres; se abrieron talleres para la enseñanza de oficios y educación práctica; y escuelas dominicales para los obreros .

\section{El hermano Miguel Febres Cordero}

La Comunidad de los Hermanos Cristianos fue establecida en Ecuador por el Presidente Gabriel García Moreno. En el año 1863 fundaron su Colegio en Cuenca, en donde fue matriculado el joven Francisco Febres Cordero, uno de los primeros alumnos, quien se formó en la filosofía lasallista, con las cualidades pedagógicas de los Hermanos Cristianos. Este joven había nacido en Cuenca el 7 de noviembre de 1854. Fueron sus padres Don Francisco Febres Cordero Montoya y Doña Ana Muñoz Cárdenas, de la aristocracia de Cuenca. Su ascendiente, el General León de Febres Cordero y Oberto; y además, era pariente del militar y político venezolano León de Febres Cordero (1795-1875), quien intervino destacadamente en la lucha por la independencia ecuatoriana, en los sucesos de Guayaquil y en la Campaña del Perú; fue Jefe del Estado Mayor del mariscal Sucre. El Libertador lo ascendió a General de División

\footnotetext{
MEZA DÍAZ, Cristhian James; JIMÉNEZ IBÁÑEZ, José Raúl; TURRIAGO ROJAS, Daniel. (2006): Historicidad, Saber y Pedagogía. Una mirada al modelo pedagógico Lasallista en Colombia, 1915-1935. Bogotá, Universidad de La Salle.

Ibídem., pp. 44-51.
} 


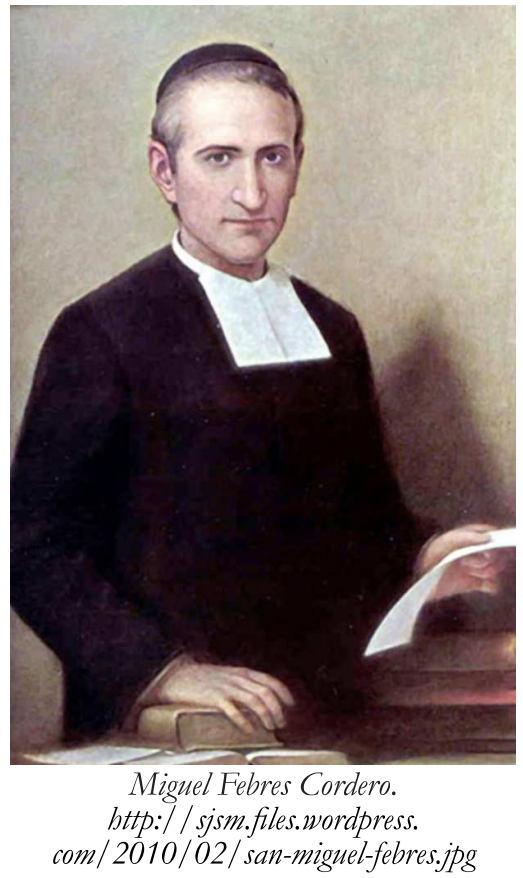

por méritos de guerra. Influyó notablemente en el Gobierno de Quito en los años de su nacimiento a la vida nacional.

El niño Francisco nació con los pies deformados, por lo cual, a los cinco años no había dado los primeros pasos. Cuenta la tradición que el niño cuando estaba observando un rosal, vio una hermosa Señora que estaba sobre las rosas. Decía a sus familiares que era una bella señora con un vestido blanco y un manto azul. Maravillados sus familiares, vieron cuando el niño se levantó y comenzó a caminar, pues estaba curado. Se consideró que era un milagro de la Virgen, con quien encontró sus fuerzas para seguir adelante. Siempre fue un devoto constante de la Virgen María .

Sus estudios los hizo en el Colegio de los Hermanos Cristianos fundado en Cuenca, cuya Comunidad fue establecida en Ecuador por el Presidente Gabriel García Moreno. El joven Francisco Febres Cordero fue uno de los primeros alumnos en dicho Colegio. Allí se formó con la filosofía lasallista, con las cualidades pedagógicas de los Hermanos Cristianos.

Se destacó en los estudios por su gran inteligencia. Sus profesores recordaban que el joven Francisco se quedaba en el Colegio preparando las lecciones y haciendo las tareas. Además, se preocupaba por ayudar a los Hermanos Cristianos en los oficios de la Comunidad. Por ello, sus Maestros le manifestaron gran aprecio ${ }^{10}$.

El estudiante Francisco Febres Cordero expresó su deseo de entrar a la Comunidad de los Hermanos Cristianos. Ante ello, su familia se opuso a sus deseos, pues eran de familia aristocrática y consideraban que los Hermanos eran pobres. Ellos querían que su hijo fuera un sacerdote y siguiera la carrera eclesiástica. Lo enviaron a otro Instituto, pero se enfermó y tuvo que volver a su casa. Por ello, la familia permitió que entrara a la Comunidad de los Hermanos Cristianos. Sin embargo, su padre que había aceptado la decisión de su esposa, no escribió a su hijo durante cinco años.

\footnotetext{
${ }^{9}$ Véase la biografía de San Miguel Febres Cordero del MUÑOZ BORRERO, Eduardo.

${ }_{10}$ Por el camino recto con los pies torcidos. Comunidad de los Hermanos Cristianos.

Ibídem., p. 44.
} 
El joven Francisco Febres Cordero entró a la Comunidad de los Hermanos Cristianos en donde tomó el nombre de Hermano Miguel Febres Cordero. De Cuenca fue trasladado a Quito. Allí se dedicó a la enseñanza y a la atención a los enfermos. Enseñaba el Catecismo a los niños de Quito y Cuenca. Fue un gran preparador de niños para la Primera Comunión durante 26 años seguidos. Además se dedicó a estudiar Idiomas, especialmente el francés, inglés, alemán, italiano y latín y se dedicó a la escritura de libros, especialmente textos escolares.

\section{G.M. Bruño y la textología}

El Hermano Miguel Febres Cordero inició su apostolado en las escuelas lasallistas de Quito. Sobresalió como profesor en la enseñanza de la Lengua y la Literatura española. Ante la carencia de un libro de texto apropiado, decidió redactarlo siguiendo sus experiencias y su didáctica como profesor de español y literatura. El texto de Lengua y Literatura española fue adoptado por el Gobierno ecuatoriano para todas las escuelas del Ecuador.

Considerando la importancia de los textos escolares para la formación educativa de las jóvenes generaciones, la Comunidad de los Hermanos Cristianos estimuló al Hermano Miguel Febres Cordero, quien para sus escritos tomó el nombre de G.M. Bruño, para que continuara escribiendo textos para la educación media y elemental. Así continuó con textos en el campo de la lírica y la filología. Con estos textos escolares, la Academia de la Lengua de Ecuador lo hizo académico y posteriormente la Real Academia Española de la Lengua le otorgó el diploma de Miembro Correspondiente. Su prestigio fue grande en las letras ecuatorianas a través de los textos escolares.

El Hermano Miguel Febres Cordero con su seudónimo de G.M. Bruño se dedicó con entusiasmo a la elaboración de textos escolares, alcanzando a redactar y publicar más de cincuenta, según la relación que se hizo de los numerosos textos publicados en Ecuador, Francia y España. Muchos de ellos fueron acogidos por diversos países hispanoamericanos, entre ellos en Colombia. El Hermano Miguel Febres Cordero era un autodidacta, destacándose por su autoinstrucción. Leía libros de numerosos autores y de diversos pueblos, aprovechando que 


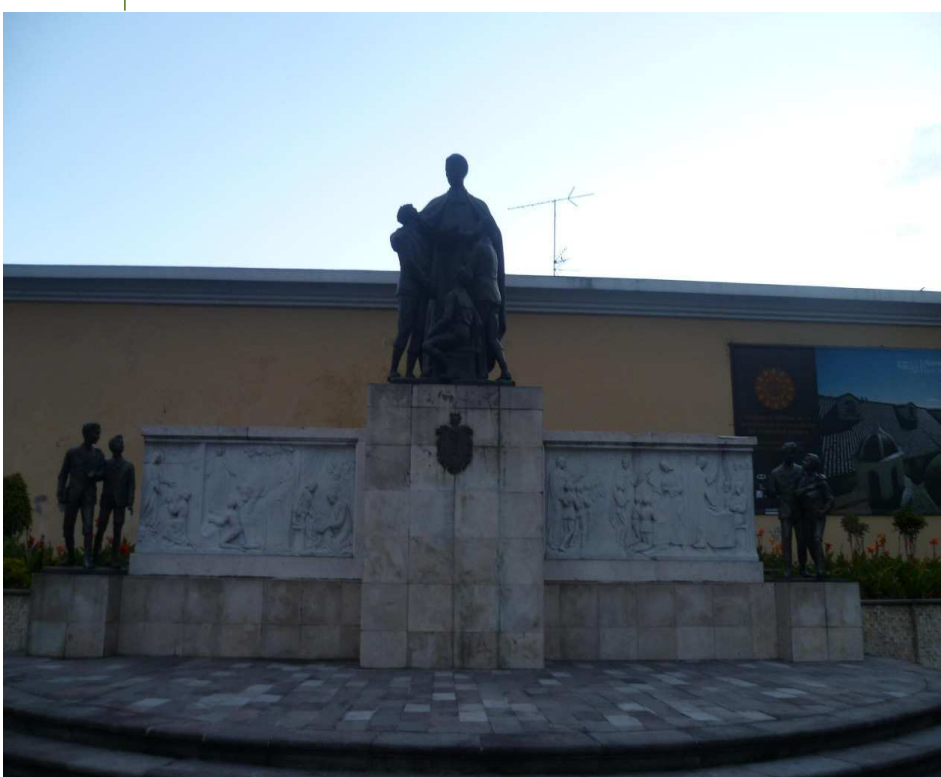

Busto de Francisco Febres Cordero. Hermano Miguel. Plaza de San Blas. Quito Ecuador. Foto por Diana Soto. 2011 conocía varios idiomas, pues uno de sus intereses fue el aprendizaje de las lenguas. Muchos de sus libros son adaptaciones de obras que habían tenido mucho interés en otros países.

En su método de estudio, el Hermano Miguel Febres Cordero escogía el texto para redactar. Seleccionaba los libros sobre el tema, de diversos autores y en varios idiomas. Siguiendo el programa escolar, hacía la síntesis de cada tema, con una temática apropiada para los escolares. Tema conciso, preciso y con una gran síntesis, según el modelo que siguen los autores de textos escolares.

El estilo en sus escritos era muy ameno, pues lo utilizó en los textos escolares como si fuera dictando sus clases. Por eso agradaban por su sencillez y por la precisión en los contenidos. El conocimiento de los temas los llevaba a su síntesis didáctica con destino a los niños y jóvenes. Cada capítulo estaba apropiado para una clase con su método, didáctica y síntesis .

Entre la serie de textos que publicó encontramos los textos de catecismo para la infancia, siendo la catequesis el campo preferido de su actividad apostólica. Lo que más le gustaba era preparar a los niños para la Primera Comunión, dedicándose a esta obra educativa cristiana hasta 1907 cuando viajó a Europa. Su contacto permanente con los niños le dio una espiritualidad en la sencillez evangélica; así les expresaba: "Sed sencillos como palomas"; por ello su devoción al Niño Jesús.

Entre sus textos de religión destacamos también los siguientes: "Catecismo de la Doctrina Cristiana" para curso medio, publicado en México, siete ediciones. "Historia Sagrada." Antiguo y Nuevo Testamento. Curso Medio, de G.M. Bruño.

${ }^{11}$ NOLEN y GOETZ. (1961): Prontuario del Escritor de Materias educativas. México, Editorial Pax-México, pp. 102-114. 
Uno de sus mayores aportes fue la redacción de textos escolares de matemáticas, que tuvieron mucha repercusión en los colegios hispanoamericanos. Destacamos entre ellos: Aritmética de G.M. Bruño, un texto para el curso medio y otro a nivel elemental. Este texto fue el que más influyó en Colombia en el siglo XX, en varias ediciones y en diversas editoriales; asimismo en otros países hispanoamericanos. En los Colegios, los profesores de matemáticas, álgebra, geometría y trigonometría, los textos que más solicitaban eran los de la Colección G.M. Bruño, con los cuales se formaron las generaciones colombianas, ecuatorianas y en general de los países hispanoamericanos.

Otros textos escolares fueron los de "Aritmética. Curso Superior". "Aritmética", Libro del Maestro. "Elementos de Geometría" " "Geometría. Curso Superior" de G.M. Bruño. El texto "Lecciones elementales de Geometría", el texto "Ejercicios y problemas, elementos de Álgebra" de G.M. Bruño. El texto "Tablas de Logaritmos"; el texto "Contabilidady Prácticas Mercantiles" de G.M. Bruño. Los textos de Botánica y Física tuvieron también mucho éxito en los estudios de educación media. Destacamos el texto "Botánica" de G.M. Bruño, editado en París. Asimismo, el texto "Curso de Física" de G.M. Bruño.

Otro texto que tuvo mucha repercusión en España e Hispanoamérica fue la "Ortografía al dictado. Tratado práctico de Ortografía", publicado por Depósitos en Madrid y Barcelona. El texto "Lecciones de Lengua Castellana. Curso superior" de G.M. Bruño, tuvo también mucha acogida y fue adoptado en numerosos Colegios de España e Hispanoamérica.

El autor de textos G.M. Bruño se generalizó en el mundo hispánico, tanto en España como en los países hispanoamericanos. Los Ministerios o Secretarías de Educación recomendaron los textos de G.M. Bruño como los más apropiados para el complemento en la instrucción educativa. En sus textos, el autor G.M. Bruño se destacó por la síntesis y la exactitud en los contenidos en toda la materia que se debía dictar, de acuerdo con los programas de las autoridades educativas. En sus contenidos, los textos de G.M. Bruño presentan un lenguaje científico accesible al nivel intelectual de los alumnos a los cuales estaba destinado ya en la educación media o en la elemental. Sus textos presentan problemas y numerosas experiencias, ejercicios y encauzamientos hacia la investigación científica para los jóvenes en su proceso de enseñanzaaprendizaje. 


\section{Su traslado a Europa, su muerte y santificación}

En el año 1907 el Hermano Miguel Febres Cordero (G.M. Bruño), después de haber trabajado en Cuenca y Quito durante más de cuarenta años, fue trasladado a Europa para que se dedicara a dirigir la publicación de libros de la Comunidad de los Hermanos Cristianos, además, la composición de textos apropiados para el estudio acelerado del español en Europa. Tras unos meses de estancia en París, el Hermano Miguel se trasladó a la Casa de los Hermanos Cristianos en Lembecq - lez - Hal (Bélgica).

Sin embargo, el clima de Bélgica con sus inviernos tan rígidos le afectó su salud; y a pesar de ello, siguió trabajando con intensidad en la edición de los libros. Ante su enfermedad, los Superiores lo trasladaron a España al Colegio de "Bonanova en Barcelona en donde el clima es más suave. A finales de enero de 1910 contrajo una pulmonía que no pudo superar. El 9 de febrero del mismo año murió el Hermano Miguel Febres Cordero en Premiá de Mar, cerca de Barcelona (España). La República de Ecuador proclamó un duelo nacional; se señalaron sus virtudes y su santidad. Después de su muerte empezaron a obtenerse milagros, con la intercesión del Hermano Miguel.

La Orden Lasallista de los Hermanos Cristianos decidió conseguir su santificación en Roma en 1913. El Hermano Paúl Joseph escribió una biografía del Hermano Miguel en francés, titulada "Un religieux Ecuaterien”. En 1923 se inició en Quito y Cuenca su proceso de beatificación. Monseñor Manuel María Pólit inició el proceso informativo sobre la vida y virtudes del Hermano Miguel. El Pontífice Pío XI firmó el Decreto de introducción a la causa de beatificación.

En enero de 1937 durante la Guerra Civil Española, los restos del Hermano Miguel fueron arrojados de la sepultura por las milicias rojas. Ante ello, el cónsul de Ecuador en Barcelona, Lic. Colón Serrano propició el traslado al suelo patrio de los restos mortales del Siervo de Dios en el Vapor Orazzio. Llegaron a Guayaquil el 5 de febrero de 1937 y fueron conducidos a Quito, la capital, en donde fueron recibidos con una acogida triunfal. Su tumba se convirtió en centro de continuas peregrinaciones. 
El milagro del Hermano Miguel a la Hermana Sor Clementina Flores Cordero, fue importante para su causa para la Beatificación. El Papa Paulo VI procedió a la Beatificación del Hermano Miguel, el 30 de octubre de 1977. Otro milagro se comprobó en la Señora Beatriz Gómez de Núñez, quien se curó de su enfermedad con la intercesión del Beato Miguel. El Papa Juan Pablo II canonizó a San Miguel Febres Cordero, en ceremonia que se celebró el 21 de octubre de 1984. El santo Miguel fue considerado un religioso culto, pero sencillo y humilde; y especialmente un catequista totalmente entregado a la obra de la evangelización. Sus virtudes fueron propias de la vida religiosa: la pureza, la pobreza y la obediencia. Se destacó su constante devoción a la

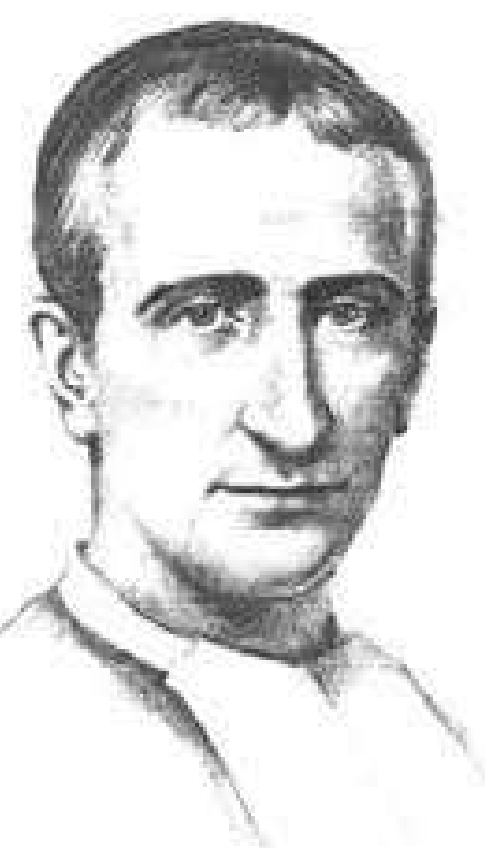

Miguel Febres Cordero. bttp:// www.cbnosf.org/images/miguel.gif Virgen. Un educador que ayudó a tantos jóvenes y niños a encontrar su vida en Jesús. Se consideró un gran educador, autor de textos y en especial, caracterizado por su obra de evangelizador. Se declaró su fiesta el 9 de febrero en el calendario eclesiástico.

Entre los autores de textos escolares en Hispanoamérica se destaca la obra de G. M. Bruño, que corresponde al autor ecuatoriano Hermano Cristiano San Miguel Febres Cordero. Su obra en la Textología escolar es una de las más importantes en Hispanoamérica, por la diversidad de las materias y la proyección en la formación de las jóvenes generaciones en los países hispanoamericanos. Su aporte es de importancia para el material didáctico en el proceso enseñanza-aprendizaje.

Los textos escolares de G.M. Bruño se caracterizaron por la síntesis, exactitud y precisión en los temas de las diversas materias 
que fueron de su interés, especialmente en las matemáticas, la geometría, el álgebra, la física, la biología, la lengua española, la ortografía, la religión, la Historia sagrada, la contabilidad y otras. El Hermano San Miguel Febres Cordero tuvo su interés por divulgar las ciencias a través de los textos escolares con un método propio de investigación pedagógica con la pedagogía católica lasallista propia de la Comunidad de los Hermanos Cristianos.

\section{FUENTES}

BRUÑO, G.M., (1911): Manual de Pedagogía para las Escuelas Católicas. París, Librería de la viuda de Ch. Bouret.

Guía de las Escuelas Cristianas. París 1903. Bogotá, Librería Stella, 1951.

Hermano Florencio Rafael y Hermano Andrés Bernardo. (1965): Los Hermanos de La Salle en Colombia. Bodas de Diamante 1890-1965. Verdadera Misión Pedagógica. Medellín, Editorial Bedout.

Hermano Herberto María. (1937): Metodología de la Religión. Escuela de Pedagogía. Lima, C. Larrabure Editor.

Manual del Catequista. (1910): Metodología de la Enseñanza de la Religión en las escuelas primarias y colegios. París, Procuraduría General.

Prospecto de la Escuela Central de Artesy Oficios. Bogotá, Noviembre de 1917.

\section{REFERENCIAS}

ABBAgnAnO, N. y Visalberghi, A. (2001): Historia de la Pedagogía. México, F.C.E.

AGATHÓN, Hermano. (1985): Las doce virtudes del buen maestro según San Juan Bautista de La Salle. Provincia Lasallista de Bogotá. Bogotá, Ediciones Samper, 1985.

AVANZINI, Guy. (1998): La pedagogía desde el siglo XVII hasta nuestros días. México, Fondo de Cultura Económica. 
BOHÓRQUEZ CASALLAS, Luis. (1965): La evolución educativa en Colombia. Bogotá, Publicaciones Cultural Colombiana.

CACUA PRADA, Antonio. (1997): Historia de la Educación en Colombia. Bogotá, Academia Colombiana de Historia. Biblioteca de Historia Nacional, vol. CL.

CONGREGACIÓN DE LOS HERMANOS DE LAS ESCUELAS CRISTIANAS. (1934): Compendio de Historia del Instituto de los Hermanos de las Escuelas Cristianas. Procure Genérale, París.

CORTS GINER, María Isabel; ÁVILA FERNÁNDEZ, Alejandro; CALDERÓN ESPAÑA, María Consolación; MONTERO PEDRERA, Ana María. (1996): Historia de la Educación. Sevilla, Gipes.

DE LA SALLE, San Juan Bautista. (1720): Guia de las Escuelas Cristianas (Manuscrito). Edición Príncipe, Distrito Lasallista de Perú.

DE LA SALLE, San Juan Bautista. (1996): Meditaciones para el Tiempo de Retiro. Bogotá, Región Latinoamericana Lasallista.

DÍAZ MEZA, Cristhian James; JIMÉNEZ IBÁÑEZ, José Raúl; TURRIAGO ROJAS, Daniel. (2006): Historicidad, Saber y Pedagogía. Una mirada al modelo pedagógico Lasallista en Colombia, 1915-1935. Bogotá, Universidad de La Salle.

EDMOND, G. (1908): Manual de Pedagogía para uso de las Escuelas Católicas de primera enseñanza. París, Procuraduría General.

GASTÓN MARÍA, Hermano. (1938): Rumbos de la Pedagogía Contemporánea. Bogotá, Editorial Centro.

GÓMEZ, M. y GÓMEZ, F. Laparticipación de la Iglesia Católica y los Hermanos de las Escuelas Cristianas en las propuestas de organización de la Instrucción pública en Colombia (1890-1948). Bogotá, Distrito Lasallista de Bogotá.

MUÑOZ BORRERO, Eduardo, H.C. Por el camino recto con los pies torcidos.

MORENO, Juan Manuel; POBLADOR Alfredo; DEL RÍO, Dionisio. (1986): Historia de la Educación. Madrid, Paraninfo, Biblioteca de Innovación Educativa.

QUICENO, H. (1988): Pedagogía Católica y Escuela Nueva en Colombia (19001935). Bogotá, Ediciones Foro Nacional por Colombia. 
RIVLIN, Harry N. y HERBERT SCHUELER. (1956): Enciclopedia de la Educación Moderna. Tomos I y II. Buenos Aires, Editorial Losada.

SÁENZ, J., Saldarriaga, et al. (1997): Mirar la infancia: Pedagogía, moral y modernidad en Colombia, 1903-1946. Bogotá, Colciencias, Foro Nacional por Colombia, Ediciones Uniandes, Editorial Universidad de Antioquia, Clío.

ZULUAGA, Olga y OSSENBACH, G. (Comp.) (2004): Modernización de los sistemas educativos iberoamericanos siglo XX. Bogotá, Cooperativa Editorial Magisterio. Tomos I y II.

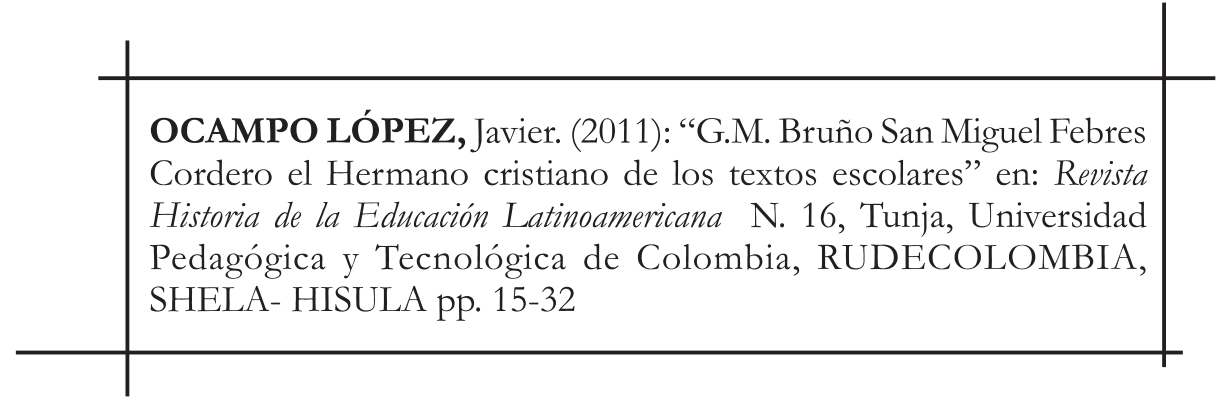

